\title{
Measuring Personality from Keyboard and Mouse Use
}

\author{
Iftikhar Ahmed Khan \\ Brunel University, Uxbridge, \\ Middlesex, UK \\ University of Engineering \\ and Technology, Peshawar \\ Pakistan \\ iftikhar.khan@brunel.ac.uk
}

\author{
Willem-Paul Brinkman \\ Delft University of \\ Technology, the \\ Netherlands \\ w.p.brinkman@tudelft.nl
}

\author{
Nick Fine \\ Brunel University, \\ Uxbridge, Middlesex, \\ UK \\ nick.fine@brunel.ac.uk
}

\author{
Robert M. Hierons \\ Brunel University, \\ Uxbridge, Middlesex, UK \\ rob.hierons@brunel.ac.uk
}

\begin{abstract}
Motivation - To measure computer users' personality from their use of keyboard and mouse.
\end{abstract}

Research approach - Two explorative studies were conducted. In first study a background application was executed on 20 participants' computers to record keys pressed and mouse clicks on an average of eight days. In a second Study 15 participants' completed a programming task in an hour while a background application recorded keys pressed and mouse clicks. Participants were asked to complete the short form of IPIP-NEO personality inventory afterwards. Pearson correlation analysis was done between participants' behaviour on keyboard, mouse events and personality ratings.

Findings/Design - The results suggest that some of the main traits and sub traits of personality can be measured from keyboard and mouse use. Significant correlations were found between personality main traits and sub traits and the use of keyboard and mouse. The maximum and minimum significant correlations were $r$ $(20)=0.62, p<=0.01$ and $r(20)=0.40, p<=0.05$ respectively in first study and were $r(15)=0.7, p<$ 0.01 and $r(15)=0.51, p<=0.05$ respectively in the second study. Personality trait activity level was found to be significantly correlated with behavioural measure 'standard deviation of average time between events' in both the studies with $r(20)=0.54, p<=0.05$ and $r$ $(15)=0.58, p<=0.05$ respectively

Research limitations/Implications - The group of participants in both studies was relatively small. There was about $40 \%$ overlap of participants in the studies.

Originality/Value - Relatively little research has focussed on personality and behaviour and specifically on the measurement of personality from the user behaviour on keyboard and mouse.

Take away message - It might be possible to measure computer users' personality from their use of keyboard

Copyright is held by the author/owner(s).

ECCE'08, September 16-19, 2008, Madeira, Portugal.

ACM 978-1-60558-399-0/08/09 and mouse.

\section{Keywords}

Personality, keyboard, mouse, behaviour, IPIP-NEO, five factor model, $\log$ file analysis, Personality measurement using computer.

\section{INTRODUCTION}

Personality is frequently measured using psychometric instruments, such as questionnaires. Whilst good retest reliability has been observed in a number of different questionnaire-based psychometric tests, the administration of them can often be time consuming. This is because the participant will usually need to complete a number of different items and score them on the appropriate scale. One issue is that typically the more items in the questionnaire, the greater the reliability of the instrument. This means that there is a tendency towards high numbers of questions and therefore longer time required for completion by participants. Likewise, another issue with questionnaire-based psychometrics is that because they can be time consuming to complete, any application that requires an understanding of the personality profile of the user is hindered by the lengthy process. In such a case gathering personality data may become an obstruction to the primary task. For example, an understanding of the user's personality profile can help personalise the user interface by providing a user interface skin that is relevant to that particular trait (Fine \& Brinkman, 2004).

This research seeks to address these limitations by developing a behaviourally based measure of personality. By removing the need for participants to become explicitly involved in the process it may be possible to measure personality whilst minimising inconvenience to the user and the obstruction to the primary task.

In order to develop a behaviourally based measure of Personality one of the underlying hypotheses in this research is that some personality traits vary as a function of interactive behaviour. There is evidence to support Personality expressed in behaviour from the literature. Brebner (1973) observed extroverts to exhibit higher levels of motor activity with their clicking buttons with a higher frequency when it caused a change in visual stimulation. Geen (1984) found that extroverts chose more intense noise levels than introverts when selecting a background level to accompany a learning task. More specifically, with 
regard to Personality and interactive behaviours, Saati, Salem and Brinkman (2005) found that extroverts tended to interact faster with the user interface than introverts, and replicates Doucet and Stelmack (1997) observations of correlations between extroversion and the speed of human movement. Such evidence suggests that Personality may be expressed in interactive behaviour and therefore provides a potential means to measure Personality by measuring interactive behaviour.

This research examines whether such behaviours can be observed as interactive behaviours, expressed through a keyboard and mouse. By capturing interactive behaviours through a log file recording of keyboard and mouse interaction, the data can then be analysed to determine whether any relationships exist between personality traits and expressed interactive behaviour. This would provide the basis for a instrument to measure personality based on interactive behaviours, facilitating measurement and determination of user personality profile without the need for explicit involvement of the participant, for example through questionnaires.

Of the various approaches to the measurement of Personality, this research adopts the dispositional approach and uses the "Big Five" model to describe Personality according to five factors: Openness to New Experience, Conscientiousness, Extroversion, Agreeableness and Neuroticism (OCEAN). A well researched and robust instrument to measure these five factors is the NEO-PIR inventory (Costa and McRae, 1992). A non-commercial alternative that measures the same five factors, with good test-retest reliability with NEO-PIR, is the IPIP-NEO inventory (Buchanan et. al. 2005), which is used in this research and described in further detail in the next sections.

\section{LOG FILES}

In this research log files were used to record keystrokes and mouse clicks. Log files have been used effectively in computer related studies for example Kukreja et al. (2005) developed a tool RUI (Recording User Interfaces) that can be used to study human robot interaction as well as human computer interaction by means of log file analysis. Haigh and Magarity (1998) used log analysis for measuring website usage. Brinkman et al. (2007) also used log files to record interaction data for usability testing of different components in a system. Log files were utilized in this research to record keyboard and mouse clicks categorically in order to find a relationship between interaction data and personality. All the capital alphabets were recorded as 'Capital Alphabet' and all short alphabets were recorded as 'Short Alphabet'. Similarly numbers were recorded as numeric and some special character like /, @ etc were recorded as 'Special Character'. The step above was to protect participants' personal data and information. Figure 1 shows an example of the log file used in this research. Four different kinds of data were being recorded in the $\log$ files and were named as:

\section{Window name}

This data was used to identify the type of application that was in use at specific event time. This data could help in identifying the participants' personality type and their keyboard and mouse behaviour with different applications. However special care was taken not to identify the names of documents that participants were working on. The application was developed to record only specific application names like Microsoft Word, Internet Explorer, and Visual Studio etc.

\section{Keyboard or mouse event}

This data was used to identify particular events. Events could either be mouse clicks or the pressing of keys. Mouse click events were indentified as "Mouse button". Key press events were further divided into two categories i.e. of 'Key Up' and of 'Key Down'.

\section{Date and time of event}

This data was used to record the date and time of the event.

\section{Category of event}

This data was used to store the category of each event that occurred. It stored 'Left' or 'Right' if the events was a mouse click and stored 'Short Alphabet', 'Capital Alphabet', 'Numeric key', 'Special Character' etc if the event was a key press event.

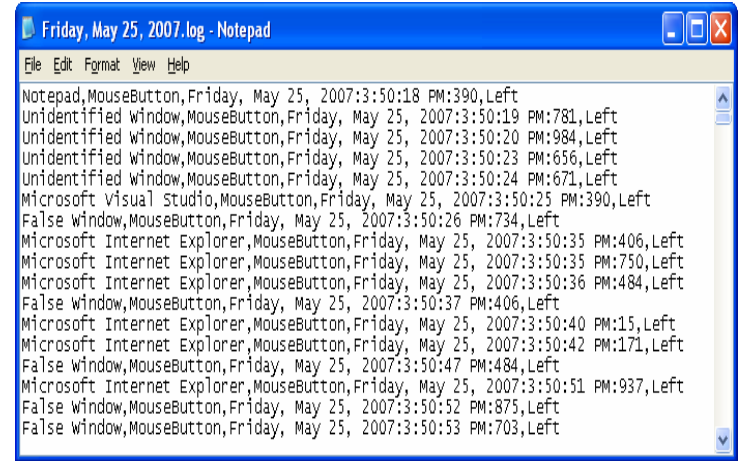

Figure 1: An example of log file

\section{STUDY 1}

\section{Material}

Both the studies reported here were part of a large study into mood, personality and behaviour and in this paper we focus only on the relation between personality and behaviour. The basic ideas of the first study were to record keyboard and mouse events in log files and correlate that with personality data. An application was developed to run in the background of the participants' computer for this purpose. The design of this application dealt ethical issues put forward by Ignatova and Brinkman (2007).

This study also used the short form of IPIP-NEO (International Personality Item Pool) an online personality test based on five factor personality model (Goldberg, 1999). The short form of IPIP-NEO is one of the versions of the personality tests, developed by 
Buchanan et al. (2005) and is available on the web ${ }^{1}$. This personality inventory contains 120 items from the original IPIP-NEO version and takes about 15-25 minutes to complete. It consists of questions about personality traits (e.g., Worry about things, Make friends easily, trust others etc)

\section{Experimental Setup}

This study was approved by the department ethical committee. All the participants were invited via personal contact or emails. Participants downloaded the application from the web to their computer and installed it. The application developed was able to detect computer user keystrokes and mouse movements and to store them in a log file. A mood rating dialog box was designed to appear every 20 minutes. Participants were able to rate their mood on this dialog and it also ensured logging of 20 minutes of interaction data for personality measurement and thus will be referred to a session in this study. A total of 86, 20minute logging interaction data sessions were planned. It was optional for participants to answer the dialog. Cohen (1992) suggested 0.10, 0.30. 0.50 effect sizes for statistically significant differences or associations for small, medium and large effects respectively. Using $\mathrm{G}^{*}$ Power analysis program by Faul et al. (2007) a sample size of 86 was calculated for a medium size effect at an alpha level of 0.05 and with a power of 0.8 . Therefore participants were instructed to answer at least 86 mood-rating dialogs to ensure adequate sample size. Application provided various functionalities such as pause logging ${ }^{2}$ for 5 minutes and 10 minutes or for variable time. It also provided exit logging ${ }^{3}$ and uninstalls the application in case participants wanted to withdraw from the study. Personality data was collected via email. Participants were asked to complete the IPIP-NEO inventory online and afterwards send the results to the experimenter.

\section{Participants}

Twenty participants completed IPIP-NEO inventory out of 26 who participated and installed application on their computer. The average age of participants was 28.5 with a standard deviation of 2.9 years. Their age range was $22-34$ years. Fifty percent of the participants classified themselves as programmers, 42 percent as expert computer users and 8 percent as medium computer users. The mean experience of computer use was 5 years with a $S D$ of 1.6 and a range

${ }^{1}$ http: //Www.personal.psu.edu/faculty/ $j / 5 / j 5 j / I P I P /$

${ }^{2}$ The participants were assured that application will not record any personal information. To further increase the trust pause logging functionality was provided allowing participants to pause logging of events for a specific time interval

${ }^{3}$ Participants were able to exit logging at any time. The application was developed to restart itself at next computer boot up process; however participants were able to restart application with out rebooting computer whenever they feel so. of 2-9 years. There were only two females among the participants.

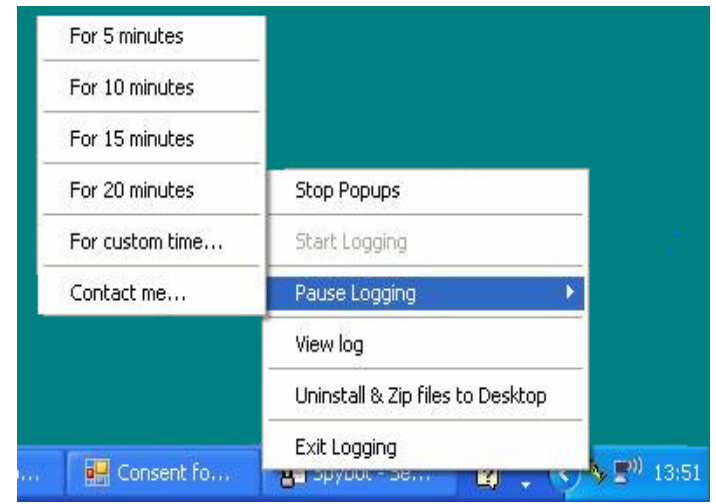

Figure 2: Application menu and its further options

\section{Data Preparation}

In the first study an average of behavioural data was calculated for every mood rating window and for each participant. The basic measures taken for each window were:

- $\quad$ Self reported valence and arousal that participants recorded in the mood rating dialog

- Total number of events around a particular mood rating

- Average time between these events

- Total windows switched

- Standard Deviation time between events

- Number of backspace and delete key events

- Number of alphabetic and numeric key events

- Number of mouse clicks

- Number of all other keys

Every participant events was monitored over an average period of 8 days. All $\log$ files created by the application in a participant computer during these days were merged to form a single log file. Each participant $\log$ file contained an average of 0.1 million lines of event recordings. An application was developed to extract the required information from the log files. The application extracted self reported mood recorded in these log files and keyboard\mouse behaviour within six minute windows around these mood ratings. The way the application extracted the data around the mood ratings within the six minute windows is illustrated in the figure 3 . 


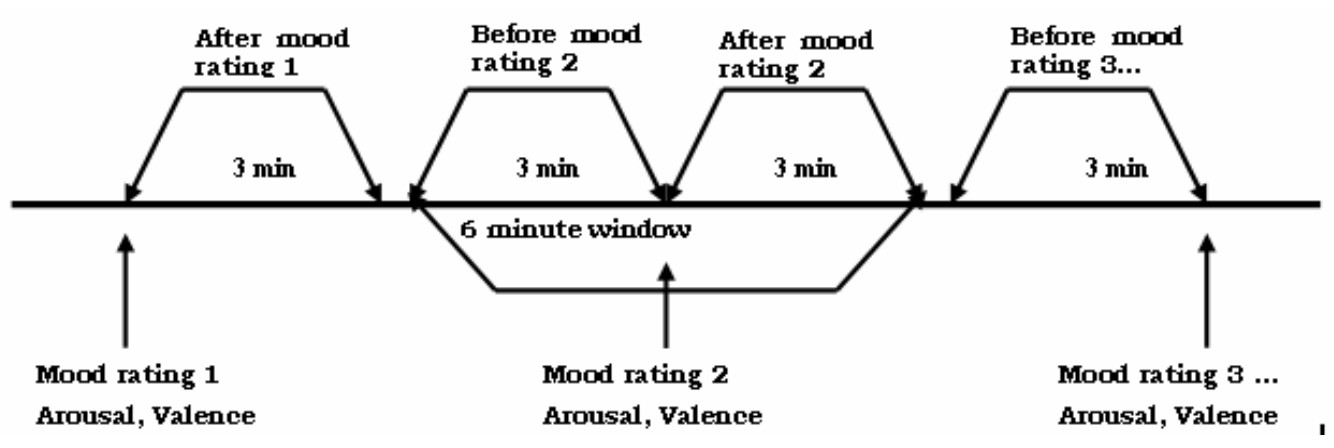

Figure 3: Window for taking events to analyze correlations between events and valence/arousal

Table 1: Correlations between Extroversion and its sub traits with that of keyboard and mouse behaviour;

\begin{tabular}{cllll}
\hline & Gregariousness & Activity Level & Excitement Seeking & Cheerfulness \\
\hline ATBE $-\mathrm{M}$ & $-.44^{*}$ & & & $-.53^{*}$ \\
ATBE-STD & $-.43^{*}$ & $.54^{*}$ & & $.41^{*}$ \\
TBE-M & $-.42^{*}$ & & $.40^{*}$ & $.41^{*}$ \\
NAKP-M & & & $.41^{*}$ & $.41^{*}$ \\
NAKP-STD & & & $.48^{*}$ & \\
NOKP-M & & & \\
NOKP-STD & & & \\
\hline
\end{tabular}

Table 2: Correlations between Agreeableness and its sub traits with that of keyboard and mouse behaviour.

\begin{tabular}{|c|c|c|c|c|c|}
\hline & Agreeableness & Trust & Morality & Cooperation & Sympathy \\
\hline ATBE-M & & $.41^{*}$ & & & \\
\hline NTWS-M & $-.56 * *$ & & $-.56 * *$ & & $-.52 *$ \\
\hline NTWS-STD & & & & & $-.48 *$ \\
\hline TBE-STD & & $.43^{*}$ & & & \\
\hline NBDKP-M & & $-.51 *$ & & & $-.43 *$ \\
\hline NBDKP-STD & $-.40 *$ & $-.40 *$ & & & $-.40 *$ \\
\hline NAKP-M & & & & $-.45^{*}$ & \\
\hline NAKP-TD & & $.47^{*}$ & & & \\
\hline
\end{tabular}

Table 3 Correlations between Conscientiousness and its sub traits with that of keyboard and mouse behaviour.

\begin{tabular}{|c|c|c|c|c|c|}
\hline & Conscientiousness & Self Efficacy & Orderliness & Dutifulness & Cautiousness \\
\hline NE-M & & & $-.60 * *$ & $-.46^{*}$ & \\
\hline NTWS-M & & & & & $-.42 *$ \\
\hline NAKP-M & & $.46^{*}$ & & & \\
\hline $\mathrm{NMC}-\mathrm{M}$ & & & & & $.44 *$ \\
\hline NMC-STD & & & & & $.52 *$ \\
\hline NOKP-M & $-.40^{*}$ & & $-.43^{*}$ & & $-.55 * *$ \\
\hline NOKP-STD & & & & & $-.56 * *$ \\
\hline
\end{tabular}

${ }^{4} \mathrm{NE}=$ Number of events; $\mathrm{ATBE}=$ Average time between Events; NTWS $=$ Number of times window switched; TBE $=$ Time between events; $\mathrm{NBDKP}=$ Number of backspace delete keys pressed; NAKP $=$ Number of alphabetic keys pressed; NMC $=$ Number of mouse clicks; NOKP $=$ Number of other keys pressed; $\mathrm{M}=$ Mean; STD $=$ Standard deviation. 
Table 4 Correlations between Neuroticism and its sub traits with that of keyboard and mouse behaviour.

\begin{tabular}{|c|c|c|c|c|}
\hline & Neuroticism & Anxiety & Self-Consciousness & Vulnerability \\
\hline ATBE (M) & & & $.43^{*}$ & \\
\hline NTWS (M) & & & $.50 *$ & \\
\hline NAKP (M) & & & $.51 *$ & \\
\hline $\mathrm{NMC}(\mathrm{M})$ & & $-.40 *$ & $-.53 *$ & \\
\hline NMC (STD) & $-.40^{*}$ & $-.40 *$ & $-.51^{*}$ & $-.41 *$ \\
\hline
\end{tabular}

Table 5: Correlations between Openness to experience and its sub traits with that of keyboard and mouse behaviour.

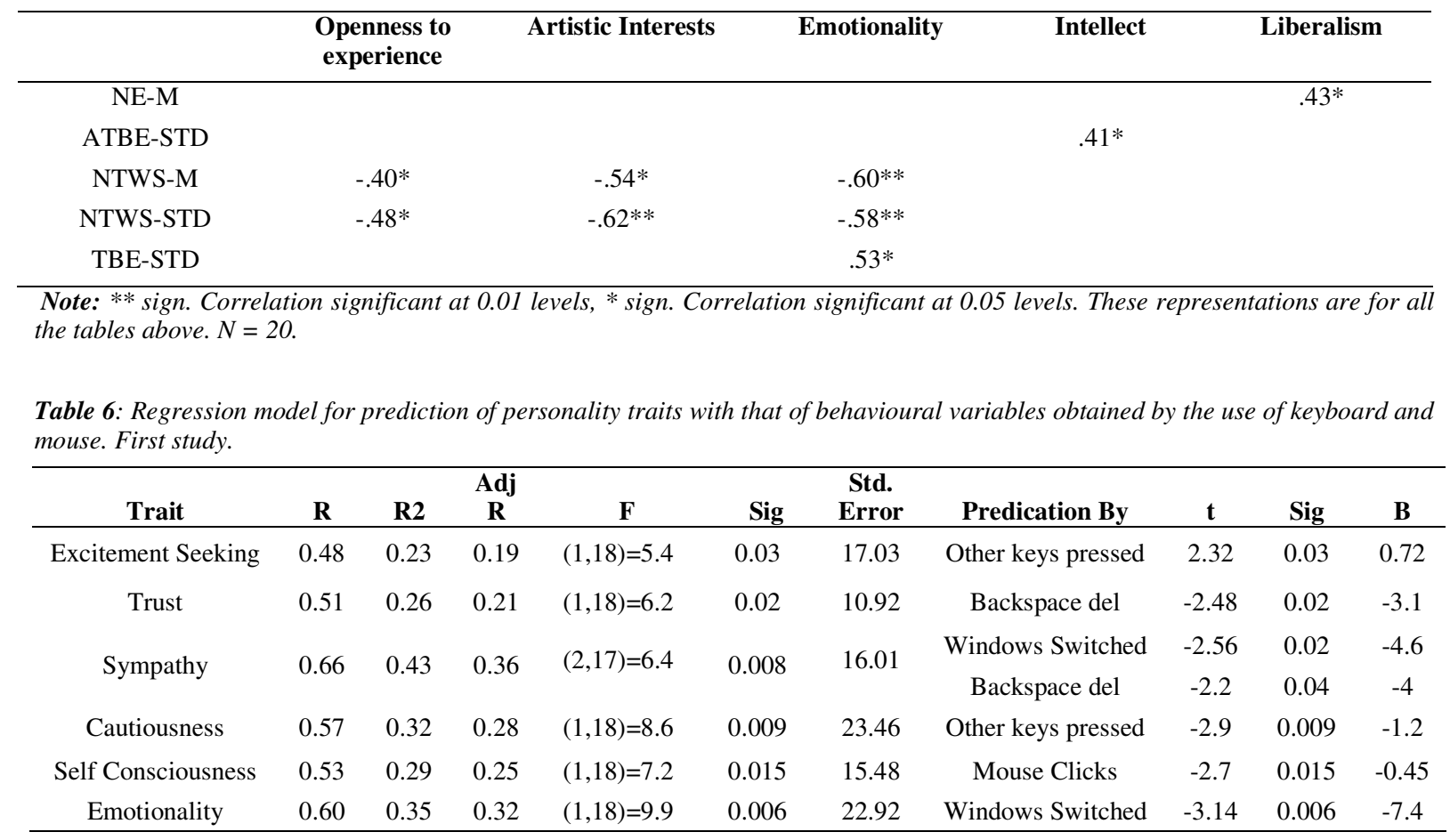

In preparation of the data we removed all data from those window slots where the number of events was less then or equal to 10 . The threshold values of 10 was selected because participants might not be active on keyboard and mouse but instead be busy in some other tasks like reading text from websites. Another filter was based on the key press and mouse click rates. All the key press and mouse click events with less then 50 milliseconds difference with previous event were filtered. Card et al. (1983) indicated that an absolute novice have a typing speed of 1000 milliseconds whereas a champion typist have an average speed of 60 milliseconds to type a character. Considering a champion typist, a lower limit of 50 milliseconds was set as filter. Similarly all the key press and mouse clicks with more then 20,000 milliseconds (20 seconds) difference with previous event were also filtered out as people waiting more than 20,000 milliseconds to click a mouse button or to type a key might be busy in some other activity like reading websites or other documents.

\section{RESULTS}

Pearson correlations were carried out between the value of each personality trait and behavioural data.
Various traits and sub traits of personality showed significant correlations with use of the keyboard and mouse. The results of the personality traits or sub traits and the behavioural variables having significant correlations are shown in the Tables 1-5.

As it emerged that various personality traits have significant correlation with behavioural data a further step was taken in the form of regression analysis to examine whether the portion of explained variance in personality trait could be increased by combining behavioural measures. Regression analysis on the significantly correlated values revealed significant prediction of some of the personality traits like Excitement seeking, Trust, Sympathy, and Emotionality etc. The results are illustrated in Table 6. Because of the difference in degree of freedom between a correlation and regression model with intercept, some of the borderline significant correlations did not resulted in a significant regression model. The results in Table 6 indicate that Excitement Seeking, Trust, Cautiousness, Self Consciousness, and Emotionality can be measured to some extent by 
behavioural data like Special keys pressed, back space delete keys pressed, Windows switched, and Mouse clicks. The Sympathy sub-trait was the only regression model with two behavioural parameters: Windows switched and backspace/delete keys pressed.

According to Cohen (1992) correlations are considered of small, medium and large size effects if $r=0.1, r=$ 0.3 to 0.49 , and $r=0.5$ or above respectively. All the correlations in the tables given above have either medium or large effects with the highest correlation being between sympathy and number of backspace delete keys pressed in a given time $(r=0.66, p=$ 0.008). Although the study found medium and large correlations, whether interaction data can be used as personality measure will depend on the level of accuracy required. For example, the explained variance of the personality traits by the regression models ranged from $23 \%$ to $43 \%$, with a standard error in the predicted value ranging from 11 to 23 points on 100point scale.

\section{STUDY 2}

\section{Objective}

From Study 1 it was found that various behavioural measures have correlations with several personality traits. However in Study 1 keyboard and mouse logging were done over several days and in natural conditions. Literature suggests that external conditions have no effect on personality and personality remains constant throughout life (Shaffer, 2000). For Study 2 it was therefore hypothesized that introduction of mood conditions caused by listening to music would still result in similar results as Study 1. Further more, the time for keyboard and mouse logging was reduced from several days to about an hour. Again it was hypothesized that with a short time span similar results would be obtained as in Study 1.

\section{Material}

The same application used in Study 1 was used to record keyboard and mouse clicks in Study 2 with some modifications. There were no sessions of 20 minutes and there was no popup dialog to appear for mood ratings. Application recorded keyboard key press and mouse clicks categorically (Original data were not recorded). Log file structure to $\log$ the data was the same as that of in Study 1. Music was used in the background to evoke different moods, for example an audio clip to elicit high arousal was Mozart's sonata (1985, track 1), and low arousal music representation was from adagio by Albinoni (1981). In addition participants were instructed to bring their own high and low arousing audio clips.

Like the previous study this study also used the short form of IPIP-NEO (International Personality Item
Pool) an online personality test based on five factor personality model (Goldberg, 1999).

In this study a number of programming tasks were allocated to participants. For these programming tasks a computer language called ALICE was used. ALICE is an object oriented language used to teach programming to children and was developed by Carnegion Mellon University. It is free to use and has some interesting aspects like visual object and visual construct and structures. It can be downloaded free from www.alice.org

\section{Experimental Setup}

This study was approved by department's ethic committee. All the participants were personally contacted. A separate room was used for the experiment without any outside disturbance. ALICE was used as a target language for participants to do tasks. All participants completed four learning tutorials present in the Alice Interface. The tutorials were about using ALICE and its various functionalities as well as introduce how to simulate stories in ALICE. Two Aesop stories ('The Hare and the tortoise' and 'the cow and the Frog') were assigned to simulate as tasks to each participant. Music was played in the background while participants completed the tasks. Participants' keyboard key press and mouse clicks were logged in a file during the task completion. Experiments took place in the individual homes within a quite room. These precautions were taken to avoid the anxiety of coming to a laboratory and because participants could be more relaxed at house. Personality data was obtained by asking participants to complete short online personality form. The participants were again asked to send results to the experimenter via email.

\section{Participants}

A total of 15 participants participated in this study. All participants were computer users with a mean age of 26 years and with a standard deviation of 3.1. The participants had an average computer usage experience of 7.3 years with a standard deviation of 2.77 years. Among participants 38 percent rated themselves as medium computer users, 31 percent rated themselves as expert computer users and 31 percent rated themselves as programmers

\section{RESULTS}

\section{Data Preparation}

In this study an average of behavioural data was calculated for the keyboard keys pressed and mouse clicks during the tasks for each participant. The basic measures were same as used in Study 1 e.g. Number of events, Average time between these events, Total windows switched, Standard deviation between events, Number of backspace and delete key events, Number of alphabetic and numeric key events, Number 
Table 7: Correlations between Extroversion, Agreeableness and their sub traits with that of keyboard and mouse behaviour.

\begin{tabular}{llllll}
\hline & \multicolumn{2}{c}{ Extroversion } & Agreeableness \\
\cline { 2 - 3 } & Extraversion & Assertiveness & Activity Level & Cheerfulness & Sympathy \\
\hline ATBE-STD & $0.7^{* *}$ & $0.66^{* *}$ & $0.58^{*}$ & $-0.59^{*}$ \\
ATBE-M & $-0.58^{*}$ & & $-0.51^{*}$ & $-0.52^{*}$ \\
NAKP-M & & & \\
NBDKP-M & & & \\
\hline Note: $* *$ sign. Correlation significant at 0.01 levels, * sign. Correlation significant at 0.05 levels $. N=15$.
\end{tabular}

Table 8: Correlations between Contentiousness, Neuroticism, and Openness to experience and their sub traits with that of keyboard and mouse behaviour.

\begin{tabular}{|c|c|c|c|c|c|c|}
\hline & \multicolumn{2}{|c|}{ Contentiousness } & \multirow{2}{*}{$\frac{\text { Neuroticism }}{\text { Immoderation }}$} & \multicolumn{3}{|c|}{ Openness to experience } \\
\hline & Orderliness & Cautiousness & & Openness to experience & Emotionality & Intellect \\
\hline NE-M & & & & & & $0.58^{*}$ \\
\hline TBE-STD & $0.57 *$ & $0.57 *$ & & & & \\
\hline NAKP-STD & & & & $-0.52 *$ & $-0.58 *$ & \\
\hline NAKP-M & $0.6^{*}$ & $0.57 *$ & & & & \\
\hline NBDKP-STD & & & & & & $0.52 *$ \\
\hline NBDKP-M & & & $0.57 *$ & & & $0.68 *$ \\
\hline NOMC-STD & & & & & & $0.62 *$ \\
\hline NOMC-M & & & & & & $0.64 * *$ \\
\hline
\end{tabular}

Note: $* *$ sign. Correlation significant at 0.01 levels, * sign. Correlation significant at 0.05 levels $. N=15$.

Table 9: Regression model for prediction of personality traits with that of behavioural variables obtained by the use of keyboard and mouse. Second study

\begin{tabular}{ccccccccccc}
\hline Trait & $\mathbf{R}$ & $\mathbf{R 2}$ & $\begin{array}{c}\text { Adj } \\
\mathbf{R}\end{array}$ & $\mathbf{F}$ & Sig & $\begin{array}{c}\text { Std. } \\
\text { Error }\end{array}$ & $\begin{array}{c}\text { Predication By } \\
\text { t }\end{array}$ & Sig & B \\
\hline Assertiveness & 0.66 & 0.43 & 0.39 & $(1,13)=10$ & 0.008 & 22.7 & $\begin{array}{c}\text { Std of average time } \\
\text { b/w events }\end{array}$ & 3.16 & 0.008 & 0.98 \\
Activity Level & 0.58 & 0.32 & 0.34 & $(1,13)=6.7$ & 0.023 & 21.3 & $\begin{array}{c}\text { Std average time } \\
\text { b/w events }\end{array}$ & -2.48 & 0.02 & -3.1 \\
Intellect & 0.68 & 0.46 & 0.42 & $(1,13)=11.2$ & 0.005 & 14.9 & Backspace/delete & 3.56 & 0.005 & 18.1 \\
\hline
\end{tabular}

of mouse clicks, and Number of all other keys. The calculated data for each participant was merged with the data from the personality questionnaire so that it formed a single row for each participant when imported to SPSS for statistical analysis.

\section{Analysis}

Pearson correlations were carried out between the value of each personality trait and behavioural data. Various personality traits and sub traits showed significant correlations with use of the keyboard and mouse in this scenario. The results of the personality traits and the behavioural variables having significant correlations are shown in the Tables 7-8. Although various behavioural variables showed significant correlations with that of personality traits, only one relation between 'standard deviation of average time between events' and 'Activity level' emerged to be significant in both studies. Activity level is a sub trait of Extraversion and is related to excitement and energy levels (Whalen, 2007). A positive correlation between activity level and the behavioural variable shows that an increase in the variation of the time between events is directly related to a increase of energy levels and excitement. These results suggest automatic measurement instrument could be developed which records interaction behaviour and provides personality information, in this case a person's activity level.

Regression analysis was carried to examine whether the portion of explained variance in a personality (sub) trait could be increased by combining behavioural measures. As can be seen in Table 9, the analysis did not result in significant multiple regression models, only single regression models. All correlations show either a medium or a large effect with the highest correlation between Intellect and number of backspace delete keys pressed in a given time $(r=0.68, p=$ 0.005).

\section{FINAL REMARKS}

The results of both studies suggest that it might be possible to measure computer users' personality from their use of keyboard and mouse. More specific, recording the standard deviation of the average time between events might give some insight into a person's activity level, a sub trait of extraversion. 
Like any empirical study, this study also has limitations. For example, the sample only included graduate and $\mathrm{PhD}$ students thus limiting the finding to that group and generalisation outside this group should be done with caution. Similarly, the $40 \%$ overlap between participants from the studies, might explain some part of the repeated observed correlations. Still sampling was done in two different environments. Also, most of participants were males, as there were only two females in the studies.

\section{Future research}

Measuring personality from the use of keyboard and mouse could be an easy and cheap approach to measure users' personality. The support for it can be strengthened by recruiting more computer users representing every walk of life.

\section{REFERENCES}

Albinoni, T.G., (1981). Adagio in G Minor for organ and strings [Recorded by SolistiVeniti, Conducted by Scimone, C.] On Albinoni's Adagios [CD]. Perivale, England Warner Classics, (1996)

Bishop-Clark., C. (1995). Cognitive style, Personality, and Computer Programming. Computer in Humnan Behaviour, Vol. 11, No. 2, pp. 241-260.

Brebner, J. (1983). A model of extraversion. Australian Journal of Psychology, 35, 349-359. Coles, G. H. (1989). SPR Presidential Address, 1988. Psychophysiology, 26, pp. 251-269.

Brinkman, W.-P., Haakama, R.. and Bouwhuis, D. G. (accepted). Component-Specific Usability testing. IEEE Transactions on Systems, Man and Cybernetics- Part A

Buchanan., T., Johnson., J. A., and Goldberg., L. R. (2005). Implementing a five-factor personality inventory for use on the internet. European $J$. of Psychological Assessment. vol. 21, pp. 115-127

Card, K.C., Moran, T.P. and Newell, A. (1983). 'The Psychology of Human-Computer Interaction', Lawrence Erlbaum Publishers, Hillsdale, New Jersey.

Cohen, J. (1992). 'Statistical Power Analysis', Current Directions in Psychological Science, Vol. 1, No. 3, pp. 98-101

Costa., P. T. Jr., and McCrae., R. R. (1992). Revised NEO Personality Inventory (NEO-PI-R) and NEO Five-factor Inventory (NEO-FFI): Interpretative report. Professional Manual, Odessa, FL, Psychological Assessment Resources

Doucet, C. and Stelmack, R.M. (1997) Movement time differentiates extraverts from introverts. Personality and Individual Differences, 23, pp. 775-786

Faul, F., Erdfelder, E., Lang, A.-G., and Buchner, A. (2007). 'G*Power 3: A flexible statistical power analysis program for the social, behavioral, and biomedical sciences'. Behavior Research Methods, 39, 175-191.
Fine, N. and Brinkman, W-P. (2004). Avoiding Average: Recording Interaction Data to Design for Specific User Groups. In M. Rauterberg Entertainment Computing - ICEC-2004 p.398401. Berlin: Springer.

Geen RG. (1984) Preferred stimulation levels in introverts and extroverts: Effects on arousal and performance. Journal of Personality and Social Psychology. Vol. 46, pp. 1303-1312.

Goldberg., L. (1999). A broad-bandwidth, public domain, personality inventory measuring the lower-level facets of several five-factor models. In Personality Psychology in Europe, Vol. 7, pp. 7-28.

Haigh, S. \& Megarity, J. (1998) Measuring Web site usage: Log file analysis. Retrieved July 2, 1999 from the World Wide Web: http://www. nlcbnc.ca/pubs/netnotes/notes57.htm

Heinstrom, J. (2000), 'The impact of personality and approaches to learning on information behaviour'. Information Research. Vol. 5, No. 3.

Ignatova., E. D. and Brinkman., W.-P. (2007). Clever tracking user behaviour over the web: enabling researchers to respect the user. Proceedings of HCI2007

Kukreja, U., Stevenson, W. E., \& Ritter, F. E. (2005) RUI-Recording User Input from interfaces under Windows, Behaviour Research Methods, Instruments, and Computer. Vol, 38. No. 4, pp. 656

Mozart, W.A. (1985). Sonata for two pianos in D major, K448 [Recorded by Perahia, M. and Lupu, R.]. On music for piano, four hands [CD]. London, Sony Classical. (1992)

Saati, B., Salem, M. \& Brinkman, W.-P., Towards customized user interface skins: investigating user personality and skin colour, Proceedings of $\mathrm{HCI}$ 2005, vol. 2, pp. 89-93, 2005.

Shaffer, D. R. (2000). Social and personality development (4th ed.). Belmont, CA: Wadsworth/Thomson Learning.

Whalen, T. (2007). A Psychological Profile of 'Defender Personality Traits, Journal of Computers, Vol. 2, No. 3, pp. 84-93. 